\title{
Characteristics of patients returning to emergency departments in
} Naples, Italy

\author{
Gabriella Di Giuseppe, Rossella Abbate, Luciana Albano, Paolo Marinelli, \\ Italo F Angelillo* and The Collaborative Research Group
}

Address: Department of Public, Clinical and Preventive Medicine, Second University of Naples, Naples, Italy

Email: Gabriella Di Giuseppe - gabrydg@libero.it; Rossella Abbate - rossella.abbate@libero.it; Luciana Albano - luciana.albano@unina2.it; Paolo Marinelli - paolo.marinelli@unina2.it; Italo F Angelillo* - italofrancesco.angelillo@unina2.it; The Collaborative Research

Group - italofrancesco.angelillo@unina2.it

* Corresponding author

Published: 2 May 2008

BMC Health Services Research 2008, 8:97 doi:10.1186/1472-6963-8-97

This article is available from: http://www.biomedcentral.com//472-6963/8/97

(c) 2008 Di Giuseppe et al; licensee BioMed Central Ltd.

This is an Open Access article distributed under the terms of the Creative Commons Attribution License (http://creativecommons.org/licenses/by/2.0), which permits unrestricted use, distribution, and reproduction in any medium, provided the original work is properly cited.
Received: 24 September 2007

Accepted: 2 May 2008

\begin{abstract}
Background: Crowding in hospital Emergency Departments (EDs) is a problem in several countries. We evaluated the number and characteristics of patients who make repeated visits to the EDs in Naples, Italy.

Methods: All patients ( $\geq 16$ years) who presented to the EDs of three randomly selected nonacademic acute care public hospitals, within randomly selected week periods, were studied. The two outcomes of interest were the re-utilization, within 72 hours, of the ED and the number of visits in the previous year.

Results: Of the 1430 sampled patients, $51.9 \%$ self-reported multiple visits in the previous year and $10.9 \%$ and $\mathrm{I} .6 \%$ used the ED for 3 and $\geq 4$ times, respectively. The number of visits in the previous year was significantly higher in those who live closer to hospital, with a more severe burden of overall comorbidity, and who were on pharmacological treatment. Overall, 72-hours return visits were found in 215 patients (I5.8\%). Patients were more likely to re-use within 72 hours the ED if younger, were not on pharmacological treatment, attended the ED more times in the previous year, were referred by a physician, arrived at the ED by car driven by other person, had problems of longer duration prior to arrival at the ED, had a surgical ED discharge diagnosis, and were admitted to the hospital.
\end{abstract}

Conclusion: The data may assist policymakers in the development and implementation of protocols to track changes in the re-utilization of the ED for the high financial impact and for the benefit of the patients.

\section{Background}

Crowding in hospital Emergency Departments (EDs) is a commonly observed problem in several industrialized countries [1-3]. Although the reasons and mechanisms are complex, the major factors are increasing volume of patients seeking medical care in ED services, lack of inpatient beds, and care for non-urgent conditions for patients who identify the ED as their usual site of care, notwithstanding the typical treatment of patients with serious illnesses or injuries. These patients typically do not receive 
any active ED intervention and could have managed adequately in other health care services, mainly the primary care facilities $[4,5]$. The decision to seek care through an ED is complex, and its explanation involves the consideration of many factors, such as patients' socio-demographic and economic characteristics, illness severity, and health service utilization behaviour.

The consequences of ED crowding include long waiting times which in turn causes delay in patient treatment and overwhelmed ED resources, and within hospital systems medical care provided by the ED represents a major component of the health care expenditures. Of particular interest is the group of patients who make many visits to ED within a brief period of time, since it is clear that frequent users consume a disproportionately large share of health care resources and hospital staff perceives them as burdensome to their workload.

Previous studies have examined the use of ED in Europe [6-8] and in the United States [9-12]. In Italy, there is a regionally-based National Health Service with an emergency medical system, providing services free of charge to all citizens, with two linked and interdependent components: pre-hospital care, provided in the community until the patient arrives at a facility capable of providing definitive care, and hospital care. No data are available in Italy and because is important to gather information on this topic from countries with this health care system, this present cross-sectional investigation sought to examine the number and characteristics of patients who make repeated visits to the EDs in Naples, Italy.

\section{Methods}

Three EDs were randomly selected among the eight nonacademic acute care public hospitals located in the geographic area of Naples (Italy). The selected hospitals have respectively 190 beds with 390,000 ED visits per year; 158 beds with 50,000 visits; and 150 beds with 74,000 visits. All patients aged 16 years or older who presented to the EDs, whether self or physician referred, from April 27 to December 17, 2006, within randomly assigned week periods were selected.

A medical interviewer, who had been previously trained and was not involved in health care, approached the patients who arrived at the ED for the current visit, indicated as index visit, after they had completed the ED registration process. After describing the purpose of the study, the interviewer collected for each patient who gave written informed consent to participate, with a brief questionnaire, the following information: socio-demographics characteristics (age, gender, marital status, educational level, living condition, employment status); distance the patient lived from the hospital; time, day of the week, and method of arrival; self-reported pre-admission performance-based measure of basic activities of daily living (BADL) using the Katz score [13]; comorbidity index of Charlson et al. [14]; pharmacological treatment(s); route of referral, symptom(s) from which the patient suffered before the presentation at the ED, duration of complaints prior to presentation; reasons for attending the ED; level of urgent care according to the Guidelines of the Canadian Emergency Department Triage and Acuity Scale [15]; and number of ED visits to acute care hospitals in the previous year. For each patient, the attending physician completed for the index visit a form about the consultation process (investigations, medical or surgical examinations, treatment received), ED discharge diagnosis, and final disposition. If a patient had already attended an ED in the previous year, the same information was obtained for every previous visit by personally asking the patient. When patient was unable to be interviewed because of his/her health status, the questionnaire was completed by relatives. A total of 27 patients were excluded because they were judged to have severe impairments, requiring immediate medical or surgical attention, that interfered with completion of the verbal assessment tools and no one else was available to provide the information.

The study was approved by the Ethics Committee of the Authors' Institution.

Non-traumatic injuries were classified anatomically (e.g., chest pain, abdominal pain) and by the most troubling symptom (e.g., shortness of breath, weakness, fever) whereas those traumatic were usually categorized by cause of injury (e.g., motor vehicle collision, fall, gunshot wound). The ED discharge diagnosis was classified according to the International Classification of Diseases (ICD)-10 and the patient was assigned to medical or surgical group on the basis of the advice of doing or not doing a surgical procedure.

The medical interviewers piloted the survey instrument with actual patients in the ED before the start of the study to evaluate validity, content, and clarity of each question and feedback was used to refine the survey instrument.

\section{Statistical analysis}

A multivariate ordered logistic and logistic regression analyses were used to determine the independent association of the potential predictors' characteristics with the following outcomes of interest: profile of the patient who attend the ED according to the number of visits in the EDs in the previous year (Model 1) and index visit as a re-utilization of the ED within 72 hours from a previous ED visit in the same or other hospital (Model 2). Two procedures were performed for these analyses. First, statistical associations between various characteristics and the out- 
comes were assessed. Univariate analysis was conducted using appropriate test statistics and those characteristics associated with the outcome variables with a $p$ value less or equal than 0.25 were included into the multivariate models. Second, multivariate models using backward elimination and stepwise selection of variables were developed and the significance level for variables entry into the final models was set at 0.2 and for removal at 0.4 . The following explanatory variables were included in the models: age (continuous, in years), gender (male $=0$, female $=1$ ), marital status (single/separated/divorced/ widowed $=0$, married $=1$ ), educational level (continuous, in years), number of other persons in the household $(0=$ $0,1=1,2=2,3=3, \geq 4=4$ ), employment status (unemployed $=0$, employed $=1)$, distance in kilometres between home and hospital $(<1=0,1-10=1,>10=2)$, total pre-admission performance-based measure of BADL (continuous), comorbidity index (continuous), and on pharmacological treatment $($ no $=0$, yes $=1)$. The following variables, related to the index visit at the $\mathrm{ED}$, were also included in Model 2: referral (self/relative $=0$, physician = 1 ), mode of transportation (categorical, car driven by patient or on foot $=0$, car driven by other person $=1$, ambulance $=2$ ), arrival time (2.00 PM-7.59 AM = 0, 8.00 AM-1.59 PM = 1), day of the week of attending (mondayfriday $=0$, saturday-sunday $=1)$, duration of presenting problem prior to arrival, in hours $(<1=0,1-24=1,>24$ $=2$ ), principal reason for attending (non-traumatic injury $=0$, traumatic injury $=1)$, therapeutic procedure $(\mathrm{s})$ received (no $=0$, yes $=1$ ), diagnostic investigation(s) received (no $=0$, yes $=1$ ), physician(s) consultation(s) received (no $=0$, yes $=1$ ), level of urgent care (categorical, non urgent $=0$, urgent $=1$, emergency $=2$ ), ED discharge diagnosis (medical $=0$, surgical $=1)$, hospital admission after the visit (no $=0$, yes $=1$ ), therapy prescription (no = 0 , yes $=1$ ), physician examination(s) prescription (no $=0$, yes $=1)$, and number of ED visits in the previous year ( 1 $=1,2=2,3=3, \geq 4=4)$. Logistic regression analysis was conducted to calculate the odds ratios (ORs) and their corresponding 95\% confidence intervals (CIs) for the association between independent variables and outcome. A two-tailed $p$-value of less or equal than 0.05 was the criterion for statistical significance. All analyses were conducted using the Stata version 8.1 software program [16].

\section{Results}

Of the 1430 patients selected, a total of 1360 agreed to participate for a response rate of $96.9 \%$ and for $14.2 \%$ of them the data was collected by proxy. Table 1 shows the characteristics of patients attending the ED according to visit frequency. More than half were male, the mean age was 46.7 years, the vast majority attended the ED during weekdays, the time of arrival was between 8 AM and 2 PM, three-quarters of visits were for non-traumatic reasons although the rate of transports for car driven by other per- son was the highest, only $8.1 \%$ were referred by physicians, and almost eight of ten cases were triaged as emergent or urgent.

A total of 706 patients (51.9\%) self-reported multiple visits to the ED in the previous year and $10.9 \%$ and $1.6 \%$ used the ED three and four or more times, respectively. Overall, 72-hours return visits were found in 215 patients (15.8\%) and respectively 11 (5.1\%) and 7 (3.3\%) of them returned three and four times in the 72 hours. In the first model the dependent variable was the number of ED visits in the previous year. Results of the stepwise multiple ordered logistic regression analysis showed that the number of visits was significantly higher in patients with shorter distance from home to hospital, in those with a more severe burden of overall comorbidity, and in those on pharmacological treatment (Model 1 in Table 2).

After multivariate logistic adjustments, the results regarding the re-utilization of the ED within 72 hours from a previous ED visit were partially in agreement with those from the unadjusted associations. Of the personal patient characteristics, only age and current pharmacological treatment were significantly associated with the outcome of interest because those younger ( OR $=0.99 ; 95 \% \mathrm{CI}$ $0.98-0.99$ ) and those not on treatment ( $\mathrm{OR}=0.56 ; 95 \%$ CI $0.36-0.86)$ were more likely to attend the ED more than once in 72 hours. The odds of more than one visit in 72 hours increased by 5 times for patients who have attended more times the ED in the previous year (95\% CI 3.89-6.45). How the patient arrived at the ED was also a significant predictor, since, when transportation by ambulance was chosen as reference category, the odds of more than one visit increased significantly by about 3 times for those who arrived by car driven by another person (95\% CI 1.21-8.58) as compared with patients who were transported by ambulance. The clinical characteristics of the patients who attended more than once in 72 hours differed from those who attend only one time. Indeed, those with problems of longer duration prior to arrival at the ED $(\mathrm{OR}=1.91 ; 95 \%$ CI $1.25-2.94 ; \mathrm{OR}=2.06$; 95\% CI $1.27-$ 3.37) and with a surgical ED discharge diagnosis (OR = 1.56; 95\% CI 1.07-2.28) attended more than once. Finally, referral and final disposition were also strong predictors of more than one ED visit in 72 hours. Indeed, patient referred by a physician was 3.12 times more likely to return compared to self or relatives referred (95\% CI 1.81-5.35) and those who return were almost twice as likely to be hospital admitted, including critical care units (95\% CI 1.06-3.14) (Model 2 in Table 2).

\section{Discussion}

This report describes the magnitude and frequency of adult patients who make repeated visits to the EDs as well the predictors of such use in an area of Italy. 
Table I: Characteristics of patients attending the Emergency Department (ED) by visit frequency

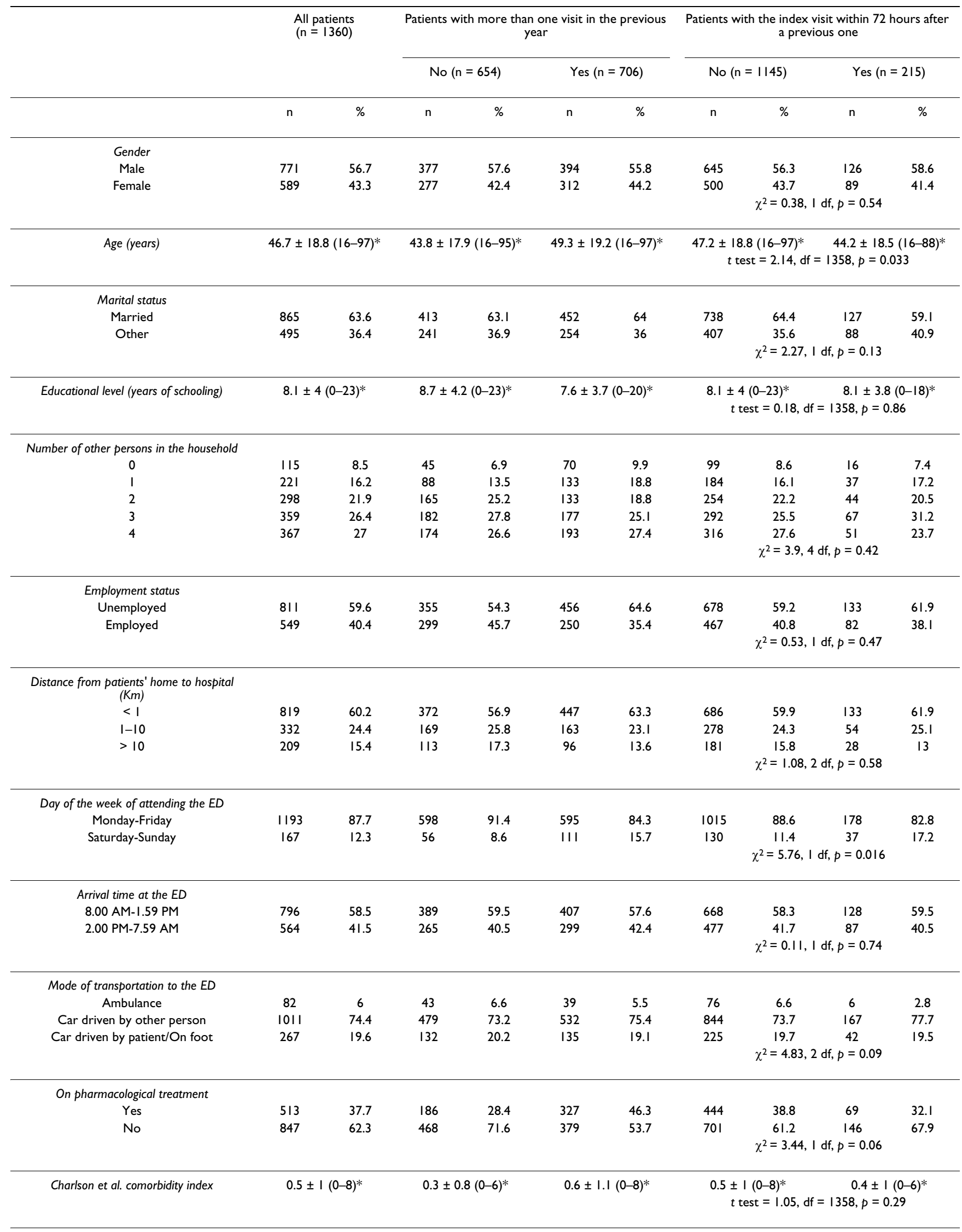


Table I: Characteristics of patients attending the Emergency Department (ED) by visit frequency (Continued)

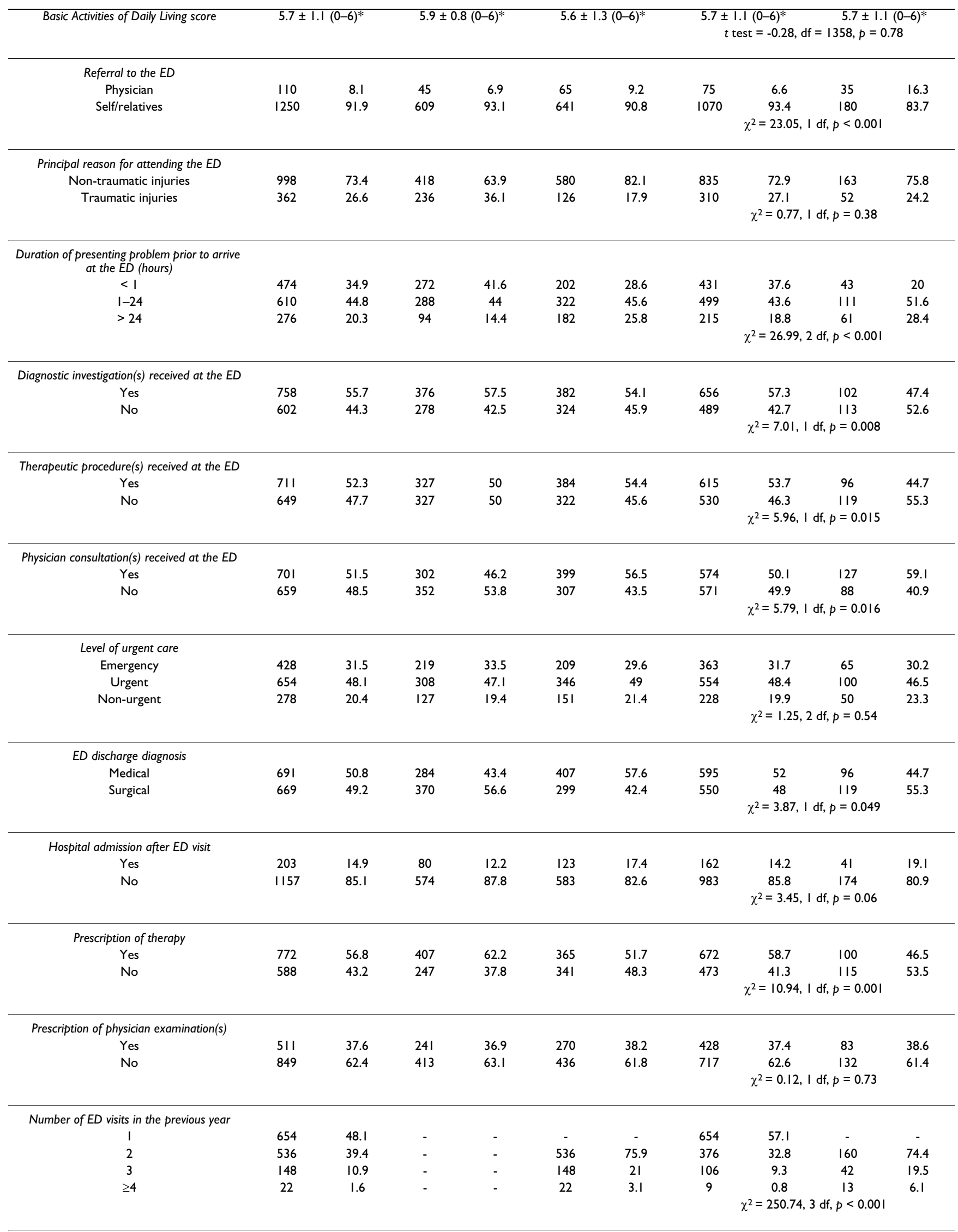

*Mean \pm Standard deviation (Range) 
Table 2: Results of the multivariate ordered logistic (1) and logistic (2) regression models

\begin{tabular}{|c|c|c|c|c|}
\hline Variable & Coeff. & SE & z & $p$ \\
\hline \multicolumn{5}{|l|}{ Model I. Number of Emergency Department visits in the previous year } \\
\hline \multicolumn{5}{|l|}{ Log likelihood $=-1347.38, \chi^{2}=99.01(6 \mathrm{df}), p<0.0001$} \\
\hline On pharmacological treatment & 0.51 & 0.12 & 4.13 & $<0.001$ \\
\hline Comorbidity index & 0.17 & 0.07 & 2.48 & 0.013 \\
\hline \multicolumn{5}{|l|}{ Distance from patients' home to hospital: } \\
\hline$>10 \mathrm{Km}^{*}$ & - & - & - & - \\
\hline$<\mathrm{I} \mathrm{Km}$ & 0.27 & 0.11 & 2.49 & 0.013 \\
\hline Educational level & -0.03 & 0.15 & -1.82 & 0.069 \\
\hline Employment status & -0.2 & 0.11 & -1.76 & 0.079 \\
\hline Basic Activities of Daily Living score & -0.09 & 0.05 & $-1.7 \mid$ & 0.088 \\
\hline Variable & OR & SE & $95 \% \mathrm{Cl}$ & $p$ \\
\hline \multicolumn{5}{|c|}{$\begin{array}{l}\text { Model 2. Index visit as a re-utilization of the Emergency Department after } 72 \text { hours from a previous } \\
\text { Emergency Department visit }\end{array}$} \\
\hline \multicolumn{5}{|c|}{ Log likelihood $=-451.26, \chi^{2}=284.73(15 \mathrm{df}), p<0.000 \mathrm{I}$} \\
\hline Number of Emergency Department visits in the previous year & 5.0 & 0.65 & $3.89-6.45$ & $<0.001$ \\
\hline Referral & 3.12 & 0.86 & $1.81-5.35$ & $<0.001$ \\
\hline \multicolumn{5}{|c|}{ Duration of presenting problem prior to arrive at the Emergency Department: } \\
\hline$<$ I hour* & 1.0 & & & \\
\hline $1-24$ hours & 1.91 & 0.42 & $1.25-2.94$ & 0.003 \\
\hline$>24$ hours & 2.06 & 0.51 & $1.27-3.37$ & 0.004 \\
\hline On pharmacological treatment & 0.56 & 0.13 & $0.36-0.86$ & 0.009 \\
\hline \multicolumn{5}{|l|}{ Mode of transportation to the Emergency Department: } \\
\hline Ambulance* & 1.0 & & & \\
\hline Car driven by other person & 3.22 & 1.61 & $1.21-8.58$ & 0.019 \\
\hline Car driven by patient/On foot & 2.54 & 1.36 & $0.89-7.24$ & 0.08 \\
\hline Emergency Department discharge diagnosis & 1.56 & 0.3 & $1.07-2.28$ & 0.022 \\
\hline Hospital admission after Emergency Department visit & 1.82 & 0.51 & $1.06-3.14$ & 0.031 \\
\hline Age & 0.99 & 0.01 & $0.98-0.99$ & 0.039 \\
\hline Therapeutic procedure(s) received at the Emergency Department & 0.69 & 0.14 & $0.47-1.01$ & 0.057 \\
\hline Physician consultation(s) received at the Emergency Department & 0.72 & 0.14 & $0.49-1.07$ & 0.1 \\
\hline Day of the week of attending the Emergency Department & 1.42 & 0.34 & $0.89-2.26$ & 0.14 \\
\hline Prescription of therapy & 0.8 & 0.15 & $0.55-1.16$ & 0.24 \\
\hline Investigation(s) received at the Emergency Department & 0.82 & 0.16 & $0.57-1.19$ & 0.31 \\
\hline
\end{tabular}

*Reference category

Comparison with other studies in the literature on re-utilization of the ED by adult patients is difficult, because there are wide differences in health care delivery system of the countries and in study design such as, for example, format of the source of data collection, time periods, and age groupings of the study population. In this study, $51.9 \%$ of patients attended an ED for more than one visit in a year and $10.9 \%$ and $1.6 \%$ attended 3 and $\geq 4$ times. Studies performed in countries with a health care system with universal access showed that in a University public Hospital in Sweden 26.9\% of patients aged 15 and above sought care more than once in the ED in a year and $4 \%$ made $\geq 4$ visits [16]; in an urban UK city over one year $3.5 \%$ and $2.2 \%$ patients made 3 and $\geq 4$ attendances, respectively [8]. Our value was considerably higher than the $31.4 \%$, collected from a statewide dataset in the United States during one fiscal year in patients of all age groups [12]. Our results were considerably lower than those observed in previously published reports. Indeed, in a National Survey of America's Families, 3\% of individuals made $\geq 3$ visits in a year [9], in a US national population-based data source, $8 \%$ of patients aged 18 and older made $\geq 4$ visits in a year [2], $4.5 \%$ in a statewide database [20], and $6 \%$ in a large rural academic medical center [21]. Our value that nearly one fifth $(15.8 \%)$ of the total attendances re-utilized an ED more than once in 72-hours was generally higher than those observed in the United States within a calendar year with values of $7.1 \%$ in an already mentioned study [21], of $3.1 \%$ to $3.7 \%$ in a nationally representative sample [12], and of $0.27 \%$ to $0.93 \%$, in two years, in a University Medical Center [10].

The investigation of the relationship between several characteristics and outcomes of interest contribute to identifying those patients who frequently re-use ED services in Italy and yielded several interesting findings. Some of the 
findings are remarkable in that they have important implications for health care workers in ED and for administrators who design systems to improve efficiency and quality of emergency care. The information of this study enables clinicians to identify patients at high risk of ED reutilization and some of these findings confirmed those of other studies, particularly that patients in fair/poor health were more likely to be frequent users $[1,2,9,22,23]$. Of note, we further found that more than one ED re-utilization within 72 hours was associated with the mode of transportation since it was significantly higher for patients arriving by independent means. A previous study has suggested that patients with potentially more serious illness or injury were more likely to be transported by ambulance [24]. Moreover, a significantly higher proportion of patients referred by physicians, usually in primary care, reused the ED within 72 hours compared with those who were self or relatives referred, suggesting a degree of clinical selection prior to attendance. This is also confirmed by the fact that no significant association has been observed between re-utilization of the ED within 72 hours and the level of urgent care. Additionally, this result is in accordance with a study conducted by some of us [4]. This inference is not surprising at all and may also be attributed to our health care system. Finally, our results also showed that those who use the ED more frequently are more likely to live closer to the hospital. Such association may suggest that at least some patients may be using the ED services for the convenience of having the hospital near home and for routine care, a finding consistent with a previous study [19]. This study suggests the need to improve communication and coordination between EDs and primary care physicians and to provide adequate patient and family education about the health care delivery system.

In interpreting the findings, potential methodological limitations should be considered. The study was conducted during the period April-December and a potential bias for seasonal variation in utilization may be present. However, in studies, conducted in a one year period in a similar geographic area, no differences have been observed according to seasons [17-19] and since differences in this one were not aspect, we are confident that there is not such potential bias. Data regarding previous ED visits were directly collected by interviewing the patient, and we cannot be absolutely certain about validity of the responses. Therefore, the results might be influenced by a recollection bias, because patients were asked about other ED visits that may have occurred up to one year before and they may have been less likely to remember with an underestimation of the true proportion of revisits. We were willing to accept this limitation, mainly because we believe that survey respondents correctly recall this information and because the principal focus was to assess the extent of patients who were making repeated visits to the ED within 72 hours from the index visit and the predictors of this re-utilization. Despite these limitations, there are several important strengths of the study. First, this is one of the few studies that collected data by interviewing patients at the ED and not by retrospective medical record reviews, with the possibility of gathering more detailed information. Second, including data from various institutions, potential biases inherent in the study of patients from a single institution have been avoided, and these data may be generalisable to all EDs. Third, data were collected over an adequate period of time. Fourth, a large number of patients agreed to participate and a such high response rate is not uncommon in cross-sectional surveys on patients attending emergency health services [7,25-28]. Fifth, multivariable analyses allowed for adjustment of several covariates.

\section{Conclusion}

In summary, the policy and management implications of these findings into the complex debate of crowding in hospital EDs are clear. The challenge for the future is that policy makers in hospital and in public health arenas should develop and implement protocols in order to track changes, not just in view of the high financial impact, but also for the benefit of the population.

\section{Competing interests}

The authors declare that they have no competing interests.

\section{Authors' contributions}

GDG and RA participated in the design of the study, collected the data, and contributed to the data analysis and interpretation; LA collected the data and contributed to the data analysis; PM participated in the design of the study and contributed to the interpretation of the data; IFA, the principal investigator, designed the study, was responsible for the data collection, statistical analysis and interpretation, and wrote the article. All authors have read and approved the final version of the manuscript.

\section{Acknowledgements}

Members of the Collaborative Research Group are: Annunziata Albano (Hospital Santa Maria della Pietà, Nola, Naples), Maurizio di Mauro (Hospital San Giovanni Bosco, Naples), Maria Anna Stingone (Hospital Evangelico Villa Betania, Naples).

Preliminary results have been presented at the $42^{\circ}$ National Congress of the Italian Public Health Association, October 27-30, 2006, Catania, Italy.

This study was supported by a grant of the Ministry of Health (Research Projects ex artt. 12 e 12 bis, Legislative decree n.502/92).

The authors wish to acknowledge the dedication of hospitals staff who voluntary participated in the survey. 


\section{References}

I. Cardin S, Afilalo M, Lang E, Collet JP, Colacone A, Tselois C, Dankoff J, Guttman A: Intervention to decrease emergency department crowding: does it have an effect on return visits and hospital readmission? Ann Emerg Med 2003, 4I: I73-I85.

2. Hunt KA, Weber EJ, Showstack JA, Colby DC, Callaham ML: Characteristics of frequent users of emergency departments. Ann Emerg Med 2006, 48: I-8.

3. Asaro PV, Lewis LM, Boxerman SB: Emergency department owercrowding: analysis of the factors of renege rate. Acad Emerg Med 2007, 14:157-162.

4. Bianco A, Pileggi C, Angelillo IF: Non-urgent visits to a hospital emergency department in Italy. Public Health 2003, I I 7:250-255.

5. Pileggi C, Nicotera G, Angelillo IF: Attendance at a hospital emergency department by drivers involved in automobile accidents in Italy. Emerg Med J 2005, 22:246-250.

6. Hansagi H, Olsson M, Sjöberg S, Tomson Y, Göransson S: Frequent use of the hospital emergency department is indicative of high use of other health care services. Ann Emerg Med 200I, 37:56I-567.

7. Byrne M, Murphy AW, Plunkett PK, McGee HM, Murray A, Bury G: Frequent attenders to an emergency department: a study of primary health care use, medical profile, and psychosocial characteristics. Ann Emerg Med 2003, 41:309-3 I8.

8. Locker TE, Baston S, Mason SM, Nicholl J: Defining frequent use of an urban emergency department. Emerg Med J 2007, 24:398-40I.

9. Zuckerman S, Shen YC: Characteristics of occasional and frequent emergency department users. Do insurance coverage and access to care matter? Med Care 2004, 42: I76-I82.

10. Martin-Gill C, Reiser RC: Risk factors for 72-hours admission to the ED. Am J Emerg Med 2004, 22:448-453.

II. Adekoya N: Patients seen in emergency departments who had a prior visit within the previous $72 \mathrm{~h}$-National Hospital Ambulatory Medical Care Survey, 2002. Public Health 2005, I 19:9|4-9|8.

12. Fuda KK, Immekus R: Frequent users of Massachusetts emergency department: a statewide analysis. Ann Emerg Med 2006, 48:9-16.

13. Katz S: Assessing self-maintenance: activities of daily living, mobility, and instrumental activities of daily living. J Am Geriatr Soc 1983, 3 1:721-727.

14. Charlson ME, Pompei P, Ales KL, McKenzie CR: A new method of classifying prognostic comorbidity in longitudinal studies: development and validation. I Chronic Dis 1987, 40:373-383.

15. Murray M, Bullard M, Grafstein E, for the CTAS and CEDIS National Working Groups: Revisions to the Canadian Emergency Department Triage and Acuity Scale: implementation guidelines. Can J Emerg Med 2004, 6:42 I-427.

16. Stata Corporation: Stata Statistical Software: Release 8.I. College Station, TX, USA 2003.

17. Angelillo IF, Ricciardi G, Nante N, Boccia A, Collaborative Group: Appropriateness of hospital utilisation in Italy. Public Health 2000, I 1 4:9-14.

18. Bianco A, Foresta MR, Greco MA, Teti V, Angelillo IF: Appropriate and inappropriate use of day-hospital care in Italy. Public Health 2002, I 1 6:368-373.

19. Pileggi C, Bianco A, Di Stasio SM, Angelillo IF: Inappropriate hospital use by patients needing urgent medical attention in Italy. Public Health 2004, I I 8:284-29I.

20. Cook LJ, Knight S, Junkins EP, Mann CN, Dean JM, Olson LM: Repeat patients to the emergency department in a statewide database. Acad Emerg Med 2004, I I:256-263.

21. Riggs JE, Davis SM, Hobbs GR, Paulson DJ, Chinnis AS, Heilman PL: Association between early returns and frequent ED visits at a rural academic medical center. Am J Emerg Med 2003, 2I:30-3I.

22. Sun BC, Burstin HR, Brennan TA: Predictors and outcomes of frequent emergency department users. Acad Emerg Med 2003, 10:320-328.

23. Weber EJ, Showstack JA, Hunt KA, Colby DC, Callaham ML: Does lack of a usual source of care or health insurance increase the likelihood of emergency department visit? Results of a national population-based study. Ann Emerg Med 2005, 45:4-I 2.
24. Ruger JP, Richter CJ, Lewis LM: Clinical and economic factors associated with ambulance use to emergency department. Acad Emerg Med 2006, 13:879-885.

25. Carret ML, Fassa AG, Kawachi I: Demand for emergency health service: factors associated with inappropriate use. $B M C$ Health Serv Res 2007, 7:131.

26. Pines JM, Garson C, Baxt WG, Rhodes KV, Shofer FS, Hollander JE: ED crowding is associated with variable perceptions of care compromise. Acad Emerg Med 2007, I 4: I I76-I I 8 I.

27. Garson C, Hollander JE, Rhodes KV, Shofer FS, Baxt WG, Pines JM: Emergency department patient preferences for boarding locations when hospitals are at full capacity. Ann Emerg Med 2008, $51: 9-12$.

28. Northington WE, Brice JH, Zou B: Use of an emergency department by nonurgent patients. Am J Emerg Med 2005, 23: I3।-I37.

\section{Pre-publication history}

The pre-publication history for this paper can be accessed here:

http://www.biomedcentral.com/1472-6963/8/97/prepub
Publish with Biomed Central and every scientist can read your work free of charge

"BioMed Central will be the most significant development for disseminating the results of biomedical research in our lifetime. "

Sir Paul Nurse, Cancer Research UK

Your research papers will be:

- available free of charge to the entire biomedical community

- peer reviewed and published immediately upon acceptance

- cited in PubMed and archived on PubMed Central

- yours - you keep the copyright

Submit your manuscript here:

http://www.biomedcentral.com/info/publishing_adv.asp 